\title{
Exponential Objects
}

\author{
Marco Riccardi \\ Via del Pero 102 \\ 54038 Montignoso \\ Italy
}

\begin{abstract}
Summary. In the first part of this article we formalize the concepts of terminal and initial object, categorical product 4] and natural transformation within a free-object category 1. In particular, we show that this definition of natural transformation is equivalent to the standard definition [13. Then we introduce the exponential object using its universal property and we show the isomorphism between the exponential object of categories and the functor category 12 .
\end{abstract}

MSC: 18A99 18A25 03B35

Keywords: exponential objects; functor category; natural transformation

MML identifier: CAT_8, version: 8.1.04 5.33.1254

The notation and terminology used in this paper have been introduced in the following articles: [2], [5], [15], [16], [17, [10], 6], 7], [11, [18, [19], 3], 8], [21], [22], [14], 20], and [9].

\section{PReliminaries}

Now we state the propositions:

(1) Let us consider a composable, associative category structure $\mathscr{C}$, and morphisms $f_{1}, f_{2}, f_{3}$ of $\mathscr{C}$. Suppose $f_{1} \triangleright f_{2}$ and $f_{2} \triangleright f_{3}$. Then $\left(f_{1} \circ f_{2}\right) \circ f_{3}=$ $f_{1} \circ\left(f_{2} \circ f_{3}\right)$.

(2) Let us consider a composable, associative category structure $\mathscr{C}$, and morphisms $f_{1}, f_{2}, f_{3}, f_{4}$ of $\mathscr{C}$. Suppose $f_{1} \triangleright f_{2}$ and $f_{2} \triangleright f_{3}$ and $f_{3} \triangleright f_{4}$. Then

(i) $\left(\left(f_{1} \circ f_{2}\right) \circ f_{3}\right) \circ f_{4}=\left(f_{1} \circ f_{2}\right) \circ\left(f_{3} \circ f_{4}\right)$, and

(ii) $\left(\left(f_{1} \circ f_{2}\right) \circ f_{3}\right) \circ f_{4}=\left(f_{1} \circ\left(f_{2} \circ f_{3}\right)\right) \circ f_{4}$, and 
(iii) $\left(\left(f_{1} \circ f_{2}\right) \circ f_{3}\right) \circ f_{4}=f_{1} \circ\left(\left(f_{2} \circ f_{3}\right) \circ f_{4}\right)$, and

(iv) $\left(\left(f_{1} \circ f_{2}\right) \circ f_{3}\right) \circ f_{4}=f_{1} \circ\left(f_{2} \circ\left(f_{3} \circ f_{4}\right)\right)$.

The theorem is a consequence of (1).

(3) Let us consider a composable category structure $\mathscr{C}$, and morphisms $f$, $f_{1}, f_{2}$ of $\mathscr{C}$. Suppose $f_{1} \triangleright f_{2}$. Then

(i) $f_{1} \circ f_{2} \triangleright f$ iff $f_{2} \triangleright f$, and

(ii) $f \triangleright f_{1} \circ f_{2}$ iff $f \triangleright f_{1}$.

(4) Let us consider a composable category structure $\mathscr{C}$ with identities, and morphisms $f_{1}, f_{2}$ of $\mathscr{C}$. Suppose $f_{1} \triangleright f_{2}$. Then

(i) if $f_{1}$ is identity, then $f_{1} \circ f_{2}=f_{2}$, and

(ii) if $f_{2}$ is identity, then $f_{1} \circ f_{2}=f_{1}$.

Proof: If $f_{1}$ is identity, then $f_{1} \circ f_{2}=f_{2}$ by [16, (6), (5), (9)].

(5) Let us consider a non empty category structure $\mathscr{C}$ with identities, and a morphism $f$ of $\mathscr{C}$. Then there exist morphisms $f_{1}, f_{2}$ of $\mathscr{C}$ such that

(i) $f_{1}$ is identity, and

(ii) $f_{2}$ is identity, and

(iii) $f_{1} \triangleright f$, and

(iv) $f \triangleright f_{2}$.

(6) Let us consider a category structure $\mathscr{C}$, objects $a, b$ of $\mathscr{C}$, and a morphism $f$ from $a$ to $b$. Suppose $\operatorname{hom}(a, b)=\{f\}$. Let us consider a morphism $g$ from $a$ to $b$. Then $f=g$.

(7) Let us consider a category structure $\mathscr{C}$, objects $a, b$ of $\mathscr{C}$, and a morphism $f$ from $a$ to $b$. Suppose $\operatorname{hom}(a, b) \neq \emptyset$ and for every morphism $g$ from $a$ to $b, f=g$. Then $\operatorname{hom}(a, b)=\{f\}$.

(8) Let us consider an object $x$, and a category structure $\mathscr{C}$. Suppose the carrier of $\mathscr{C}=\{x\}$ and the composition of $\mathscr{C}=\{\langle\langle x, x\rangle, x\rangle\}$. Then $\mathscr{C}$ is a non empty category.

Proof: For every object $y, y \in$ the composition of the discrete category of $\{x\}$ iff $y \in\{\langle\langle x, x\rangle, x\rangle\}$ by [22, (2)], [9, (29)], [15, (24)], (4).

(9) Let us consider categories $\mathscr{C}_{1}, \mathscr{C}_{2}$, and a functor $\mathcal{F}$ from $\mathscr{C}_{1}$ to $\mathscr{C}_{2}$. If $\mathcal{F}$ is isomorphism, then $\mathcal{F}$ is bijective.

(10) Let us consider composable category structures $\mathscr{C}_{1}, \mathscr{C}_{2}, \mathscr{C}_{3}$ with identities. Suppose $\mathscr{C}_{1} \cong \mathscr{C}_{2}$ and $\mathscr{C}_{2} \cong \mathscr{C}_{3}$. Then $\mathscr{C}_{1} \cong \mathscr{C}_{3}$.

(11) Let us consider categories $\mathscr{C}_{1}, \mathscr{C}_{2}$. Suppose $\mathscr{C}_{1} \cong \mathscr{C}_{2}$. Then $\mathscr{C}_{1}$ is empty if and only if $\mathscr{C}_{2}$ is empty. 
Let $\mathscr{C}_{1}$ be an empty category structure with identities and $\mathscr{C}_{2}$ be category structure with identities. Note that every functor from $\mathscr{C}_{1}$ to $\mathscr{C}_{2}$ is covariant.

Now we state the propositions:

(12) Let us consider category structures $\mathscr{C}_{1}, \mathscr{C}_{2}$ with identities, a morphism $f$ of $\mathscr{C}_{1}$, and a functor $\mathcal{F}$ from $\mathscr{C}_{1}$ to $\mathscr{C}_{2}$. Suppose $\mathcal{F}$ is covariant and $f$ is identity. Then $\mathcal{F}(f)$ is identity.

(13) Let us consider category structures $\mathscr{C}_{1}, \mathscr{C}_{2}$ with identities, morphisms $f_{1}, f_{2}$ of $\mathscr{C}_{1}$, and a functor $\mathcal{F}$ from $\mathscr{C}_{1}$ to $\mathscr{C}_{2}$. Suppose $\mathcal{F}$ is covariant and $f_{1} \triangleright f_{2}$. Then

(i) $\mathcal{F}\left(f_{1}\right) \triangleright \mathcal{F}\left(f_{2}\right)$, and

(ii) $\mathcal{F}\left(f_{1} \circ f_{2}\right)=\mathcal{F}\left(f_{1}\right) \circ \mathcal{F}\left(f_{2}\right)$.

(14) Let us consider an object-category $\mathscr{C}$, a morphism $f$ of $\mathscr{C}$, and a morphism $g$ of alter $\mathscr{C}$. Suppose $f=g$. Then

(i) $\operatorname{dom} g=\operatorname{id}_{\operatorname{dom} f}$, and

(ii) $\operatorname{cod} g=\operatorname{id}_{\operatorname{cod} f}$.

Proof: Consider $d_{1}$ being a morphism of alter $\mathscr{C}$ such that $\operatorname{dom} g=d_{1}$ and $g \triangleright d_{1}$ and $d_{1}$ is identity. Reconsider $d_{2}=\operatorname{id}_{\operatorname{dom} f}$ as a morphism of alter $\mathscr{C}$. For every morphism $f_{1}$ of alter $\mathscr{C}$ such that $f_{1} \triangleright d_{2}$ holds $f_{1} \circ d_{2}=$ $f_{1}$ by [15, (40)], [5, (22)]. Consider $c_{1}$ being a morphism of alter $\mathscr{C}$ such that $\operatorname{cod} g=c_{1}$ and $c_{1} \triangleright g$ and $c_{1}$ is identity. Reconsider $c_{2}=\operatorname{id}_{\operatorname{cod} f}$ as a morphism of alter $\mathscr{C}$. For every morphism $f_{1}$ of alter $\mathscr{C}$ such that $f_{1} \triangleright c_{2}$ holds $f_{1} \circ c_{2}=f_{1}$ by [15, (40)], [5. (22)].

(15) There exists a morphism $f$ of $\mathbf{1}$ such that

(i) $f$ is identity, and

(ii) $\mathrm{Ob} \mathbf{1}=\{f\}$, and

(iii) Mor $\mathbf{1}=\{f\}$.

Proof: Consider $\mathscr{C}$ being a strict, a preorder category such that $\mathrm{Ob} \mathscr{C}=1$ and for every objects $o_{1}, o_{2}$ of $\mathscr{C}$ such that $o_{1} \in o_{2}$ holds hom $\left(o_{1}, o_{2}\right)=$ $\left\{\left\langle o_{1}, o_{2}\right\rangle\right\}$ and $\operatorname{RelOb} \mathscr{C}=\subseteq_{1}$ and Mor $\mathscr{C}=1 \cup\left\{\left\langle o_{1}, o_{2}\right\rangle\right.$, where $o_{1}, o_{2}$ are elements of $\left.1: o_{1} \in o_{2}\right\}$. Consider $\mathcal{F}$ being a functor from $\mathscr{C}$ to $\mathbf{1}, \mathcal{G}$ being a functor from 1 to $\mathscr{C}$ such that $\mathcal{F}$ is covariant and $\mathcal{G}$ is covariant and $\mathcal{G} \circ \mathcal{F}=\operatorname{id}_{\mathscr{C}}$ and $\mathcal{F} \circ \mathcal{G}=\mathrm{id}_{\mathbf{1}}$. Reconsider $g=0$ as a morphism of $\mathscr{C}$. Set $f=\mathcal{F}(g)$. Consider $x$ being an object such that $\mathrm{Ob} \mathbf{1}=\{x\}$. For every object $x, x \in$ Mor 1 iff $x \in\{f\}$ by [15, (22)], [6, (18)], [15, (34)], [2, (49)].

(16) Let us consider a non empty category $\mathscr{C}$, and morphisms $f_{1}, f_{2}$ of $\mathscr{C}$. If $\mathcal{M}_{\mathrm{f}_{1}}=\mathcal{M}_{\mathrm{f}_{2}}$, then $f_{1}=f_{2}$. 
(17) Let us consider a non empty category $\mathscr{C}$, covariant functors $\mathcal{F}_{1}, \mathcal{F}_{2}$ from 2 to $\mathscr{C}$, and a morphism $f$ of $\mathbf{2}$. Suppose $f$ is not identity and $\mathcal{F}_{1}(f)=\mathcal{F}_{2}(f)$. Then $\mathcal{F}_{1}=\mathcal{F}_{2}$.

Proof: Consider $f_{1}$ being a morphism of $\mathbf{2}$ such that $f_{1}$ is not identity and $\operatorname{Ob} \mathbf{2}=\left\{\operatorname{dom} f_{1}, \operatorname{cod} f_{1}\right\}$ and Mor $\mathbf{2}=\left\{\operatorname{dom} f_{1}, \operatorname{cod} f_{1}, f_{1}\right\}$ and $\operatorname{dom} f_{1}$, $\operatorname{cod} f_{1}, f_{1}$ are mutually different. For every object $x$ such that $x \in \operatorname{dom} \mathcal{F}_{1}$ holds $\mathcal{F}_{1}(x)=\mathcal{F}_{2}(x)$ by [15, (22), (32)].

(18) There exist morphisms $f_{1}, f_{2}$ of $\mathbf{3}$ such that

(i) $f_{1}$ is not identity, and

(ii) $f_{2}$ is not identity, and

(iii) $\operatorname{cod} f_{1}=\operatorname{dom} f_{2}$, and

(iv) $\mathrm{Ob} \mathbf{3}=\left\{\operatorname{dom} f_{1}, \operatorname{cod} f_{1}, \operatorname{cod} f_{2}\right\}$, and

(v) Mor $\mathbf{3}=\left\{\operatorname{dom} f_{1}, \operatorname{cod} f_{1}, \operatorname{cod} f_{2}, f_{1}, f_{2}, f_{2} \circ f_{1}\right\}$, and

(vi) $\operatorname{dom} f_{1}, \operatorname{cod} f_{1}, \operatorname{cod} f_{2}, f_{1}, f_{2}, f_{2} \circ f_{1}$ are mutually different.

Proof: Consider $\mathscr{C}$ being a strict, a preorder category such that $\mathrm{Ob} \mathscr{C}=3$ and for every objects $o_{1}, o_{2}$ of $\mathscr{C}$ such that $o_{1} \in o_{2} \operatorname{holds} \operatorname{hom}\left(o_{1}, o_{2}\right)=$ $\left\{\left\langle o_{1}, o_{2}\right\rangle\right\}$ and RelOb $\mathscr{C}=\subseteq_{3}$ and Mor $\mathscr{C}=3 \cup\left\{\left\langle o_{1}, o_{2}\right\rangle\right.$, where $o_{1}, o_{2}$ are elements of $\left.3: o_{1} \in o_{2}\right\}$. Consider $\mathcal{F}$ being a functor from $\mathscr{C}$ to $\mathbf{3}, \mathcal{G}$ being a functor from 3 to $\mathscr{C}$ such that $\mathcal{F}$ is covariant and $\mathcal{G}$ is covariant and $\mathcal{G} \circ \mathcal{F}=\operatorname{id}_{\mathscr{C}}$ and $\mathcal{F} \circ \mathcal{G}=\operatorname{id}_{\mathbf{3}}$. Reconsider $g_{1}=\langle 0,1\rangle$ as a morphism of $\mathscr{C} \cdot g_{1}$ is not identity by [15, (22)]. Set $f_{1}=\mathcal{F}\left(g_{1}\right)$. Reconsider $g_{2}=\langle 1$, $2\rangle$ as a morphism of $\mathscr{C} . g_{2}$ is not identity by [15, (22)]. Set $f_{2}=\mathcal{F}\left(g_{2}\right)$. $f_{1}$ is not identity by [6, (18)], [15, (34)]. $f_{2}$ is not identity by [6, (18)], [15. (34)]. For every object $x, x \in \operatorname{Ob} 3$ iff $x \in\left\{\operatorname{dom} f_{1}, \operatorname{cod} f_{1}, \operatorname{cod} f_{2}\right\}$ by [15, (34)], 66, (18)], [15, (22)], [2, (51)]. For every object $x, x \in$ Mor 3 iff $x \in\left\{\operatorname{dom} f_{1}, \operatorname{cod} f_{1}, \operatorname{cod} f_{2}, f_{1}, f_{2}, f_{2} \circ f_{1}\right\}$ by [15, (22)], [6, (18)], [15, (34)], [2, (51), (49), (50)]. $g_{2} \circ g_{1}$ is not identity by [15, (22)]. $f_{2} \circ f_{1}$ is not identity by [6, (18)], [15, (34)]. $\mathcal{F}$ is bijective.

Let $\mathscr{C}$ be a non empty category and $f_{1}, f_{2}$ be morphisms of $\mathscr{C}$. Assume $f_{1} \triangleright f_{2}$. The functor $\mathcal{C}_{f_{1}, f_{2}}$ yielding a covariant functor from 3 to $\mathscr{C}$ is defined by

(Def. 1) for every morphisms $g_{1}, g_{2}$ of $\mathbf{3}$ such that $g_{1} \triangleright g_{2}$ and $g_{1}$ is not identity and $g_{2}$ is not identity holds $i t\left(g_{1}\right)=f_{1}$ and $i t\left(g_{2}\right)=f_{2}$. 


\section{Terminal Objects}

Let $\mathscr{C}$ be a category structure and $a$ be an object of $\mathscr{C}$. We say that $a$ is terminal if and only if

(Def. 2) for every object $b$ of $\mathscr{C}, \operatorname{hom}(b, a) \neq \emptyset$ and there exists a morphism $f$ from $b$ to $a$ such that for every morphism $g$ from $b$ to $a, f=g$.

Now we state the propositions:

(19) Let us consider a category structure $\mathscr{C}$, and an object $b$ of $\mathscr{C}$. Then $b$ is terminal if and only if for every object $a$ of $\mathscr{C}$, there exists a morphism $f$ from $a$ to $b$ such that $\operatorname{hom}(a, b)=\{f\}$. The theorem is a consequence of (7) and (6).

(20) Let us consider category structure $\mathscr{C}$ with identities, and an object $a$ of $\mathscr{C}$. Suppose $a$ is terminal. Let us consider a morphism $h$ from $a$ to $a$. Then id- $a=h$.

(21) Let us consider a composable category structure $\mathscr{C}$ with identities, and objects $a, b$ of $\mathscr{C}$. If $a$ is terminal and $b$ is terminal, then $a$ and $b$ are isomorphic. The theorem is a consequence of (20).

(22) Let us consider a category $\mathscr{C}$, and objects $a, b$ of $\mathscr{C}$. If $b$ is terminal and $a$ and $b$ are isomorphic, then $a$ is terminal.

(23) Let us consider a composable category structure $\mathscr{C}$ with identities, objects $a, b$ of $\mathscr{C}$, and a morphism $f$ from $a$ to $b$. Suppose $\operatorname{hom}(a, b) \neq \emptyset$ and $a$ is terminal. Then $f$ is monomorphic.

Let $\mathscr{C}$ be a category. We say that $\mathscr{C}$ has terminal objects if and only if

(Def. 3) there exists an object $a$ of $\mathscr{C}$ such that $a$ is terminal.

Now we state the proposition:

(24) 1 has terminal objects.

Proof: Consider $f$ being a morphism of 1 such that $f$ is identity and $\mathrm{Ob} \mathbf{1}=\{f\}$ and Mor $\mathbf{1}=\{f\}$. For every objects $a, b$ of $\mathbf{1}$, every morphism of 1 is a morphism from $a$ to $b$ by [16, (20)].

One can verify that there exists a category which has terminal objects.

Let $\mathscr{C}$ be a category. We say that $\mathscr{C}$ is terminal if and only if

(Def. 4) for every category $\mathscr{B}$, there exists a functor $\mathcal{F}$ from $\mathscr{B}$ to $\mathscr{C}$ such that $\mathcal{F}$ is covariant and for every functor $\mathcal{G}$ from $\mathscr{B}$ to $\mathscr{C}$ such that $\mathcal{G}$ is covariant holds $\mathcal{F}=\mathcal{G}$.

Let us note that $\mathbf{1}$ is non empty and terminal and there exists a category which is strict, non empty, and terminal and there exists a category which is strict and non terminal.

Now we state the propositions: 
(25) Let us consider terminal categories $\mathscr{C}, \mathscr{D}$. Then $\mathscr{C} \cong \mathscr{D}$.

Proof: There exists a functor $\mathcal{F}$ from $\mathscr{C}$ to $\mathscr{D}$ and there exists a functor $\mathcal{G}$ from $\mathscr{D}$ to $\mathscr{C}$ such that $\mathcal{F}$ is covariant and $\mathcal{G}$ is covariant and $\mathcal{G} \circ \mathcal{F}=\mathrm{id} \mathscr{C}$ and $\mathcal{F} \circ \mathcal{G}=\mathrm{id}_{\mathscr{D}}$ by [15, (35)].

(26) Let us consider categories $\mathscr{C}, \mathscr{D}$. Suppose $\mathscr{C}$ is terminal and $\mathscr{C} \cong \mathscr{D}$. Then $\mathscr{D}$ is terminal.

Proof: Consider $\mathcal{F}$ being a functor from $\mathscr{C}$ to $\mathscr{D}, \mathcal{G}$ being a functor from $\mathscr{D}$ to $\mathscr{C}$ such that $\mathcal{F}$ is covariant and $\mathcal{G}$ is covariant and $\mathcal{G} \circ \mathcal{F}=\mathrm{id}_{\mathscr{C}}$ and $\mathcal{F} \circ \mathcal{G}=\mathrm{id}_{\mathscr{D}}$. Consider $\mathcal{F}_{1}$ being a functor from $\mathscr{B}$ to $\mathscr{C}$ such that $\mathcal{F}_{1}$ is covariant and for every functor $\mathcal{G}$ from $\mathscr{B}$ to $\mathscr{C}$ such that $\mathcal{G}$ is covariant holds $\mathcal{F}_{1}=\mathcal{G}$. Set $\mathcal{F}_{2}=\mathcal{F} \circ \mathcal{F}_{1}$. For every functor $\mathcal{G}_{1}$ from $\mathscr{B}$ to $\mathscr{D}$ such that $\mathcal{G}_{1}$ is covariant holds $\mathcal{F}_{2}=\mathcal{G}_{1}$ by [15, (35)], [16, (10), (11)].

(27) Let us consider a category $\mathscr{C}$. Then $\mathscr{C}$ is non empty and trivial if and only if $\mathscr{C} \cong \mathbf{1}$. The theorem is a consequence of (15), (4), and (26).

(28) Let us consider non empty categories $\mathscr{C}, \mathscr{D}$. Suppose $\mathscr{C}$ is trivial and $\mathscr{D}$ is trivial. Then $\mathscr{C} \cong \mathscr{D}$. The theorem is a consequence of (27) and (10).

Note that every category which is non empty and trivial is also terminal and every category which is terminal is also non empty and trivial.

Let $\mathscr{C}$ be a category. The functor $\mathscr{C} \rightarrow \mathbf{1}$ yielding a covariant functor from $\mathscr{C}$ to 1 is defined by

(Def. 5) not contradiction.

Now we state the proposition:

(29) Let us consider categories $\mathscr{C}, \mathscr{C}_{1}, \mathscr{C}_{2}$, a functor $\mathcal{F}_{1}$ from $\mathscr{C}$ to $\mathscr{C}_{1}$, and a functor $\mathcal{F}_{2}$ from $\mathscr{C}$ to $\mathscr{C}_{2}$. Suppose $\mathcal{F}_{1}$ is covariant and $\mathcal{F}_{2}$ is covariant. Then $\mathscr{C}_{1} \rightarrow \mathbf{1} \circ \mathcal{F}_{1}=\mathscr{C}_{2} \rightarrow \mathbf{1} \circ \mathcal{F}_{2}$.

\section{Initial OBJects}

Let $\mathscr{C}$ be a category structure and $a$ be an object of $\mathscr{C}$. We say that $a$ is initial if and only if

(Def. 6) for every object $b$ of $\mathscr{C}, \operatorname{hom}(a, b) \neq \emptyset$ and there exists a morphism $f$ from $a$ to $b$ such that for every morphism $g$ from $a$ to $b, f=g$.

Now we state the propositions:

(30) Let us consider a category structure $\mathscr{C}$, and an object $b$ of $\mathscr{C}$. Then $b$ is initial if and only if for every object $a$ of $\mathscr{C}$, there exists a morphism $f$ from $b$ to $a$ such that $\operatorname{hom}(b, a)=\{f\}$. The theorem is a consequence of (7) and (6). 
(31) Let us consider category structure $\mathscr{C}$ with identities, and an object $a$ of $\mathscr{C}$. Suppose $a$ is initial. Let us consider a morphism $h$ from $a$ to $a$. Then id- $a=h$.

(32) Let us consider a composable category structure $\mathscr{C}$ with identities, and objects $a, b$ of $\mathscr{C}$. If $a$ is initial and $b$ is initial, then $a$ and $b$ are isomorphic. The theorem is a consequence of (31).

(33) Let us consider a category $\mathscr{C}$, and objects $a, b$ of $\mathscr{C}$. If $b$ is initial and $b$ and $a$ are isomorphic, then $a$ is initial.

(34) Let us consider a composable category structure $\mathscr{C}$ with identities, objects $a, b$ of $\mathscr{C}$, and a morphism $f$ from $a$ to $b$. Suppose $\operatorname{hom}(a, b) \neq \emptyset$ and $b$ is initial. Then $f$ is epimorphic.

Let $\mathscr{C}$ be a category. We say that $\mathscr{C}$ has initial objects if and only if

(Def. 7) there exists an object $a$ of $\mathscr{C}$ such that $a$ is initial.

Now we state the proposition:

(35) 1 has initial objects.

Proof: Consider $f$ being a morphism of 1 such that $f$ is identity and $\mathrm{Ob} \mathbf{1}=\{f\}$ and Mor $\mathbf{1}=\{f\}$. For every objects $a, b$ of $\mathbf{1}$, every morphism of $\mathbf{1}$ is a morphism from $a$ to $b$ by [16, (20)].

Let us note that there exists a category which has initial objects.

Let $\mathscr{C}$ be a category. We say that $\mathscr{C}$ is initial if and only if

(Def. 8) for every category $\mathscr{C}_{1}$, there exists a functor $\mathcal{F}$ from $\mathscr{C}$ to $\mathscr{C}_{1}$ such that $\mathcal{F}$ is covariant and for every functor $\mathcal{F}_{1}$ from $\mathscr{C}$ to $\mathscr{C}_{1}$ such that $\mathcal{F}_{1}$ is covariant holds $\mathcal{F}=\mathcal{F}_{1}$.

One can verify that $\mathbf{0}$ is empty and initial and there exists a category which is strict, empty, and initial and there exists a category which is strict and non initial.

Now we state the propositions:

(36) Let us consider initial categories $\mathscr{C}, \mathscr{D}$. Then $\mathscr{C} \cong \mathscr{D}$.

Proof: There exists a functor $\mathcal{F}$ from $\mathscr{C}$ to $\mathscr{D}$ and there exists a functor $\mathcal{G}$ from $\mathscr{D}$ to $\mathscr{C}$ such that $\mathcal{F}$ is covariant and $\mathcal{G}$ is covariant and $\mathcal{G} \circ \mathcal{F}=\mathrm{id}_{\mathscr{C}}$ and $\mathcal{F} \circ \mathcal{G}=$ id $\mathscr{D}_{\text {by }}[15,(35)]$.

(37) Let us consider categories $\mathscr{C}, \mathscr{D}$. Suppose $\mathscr{C}$ is initial and $\mathscr{C} \cong \mathscr{D}$. Then $\mathscr{D}$ is initial.

Proof: Consider $\mathcal{F}$ being a functor from $\mathscr{C}$ to $\mathscr{D}, \mathcal{G}$ being a functor from $\mathscr{D}$ to $\mathscr{C}$ such that $\mathcal{F}$ is covariant and $\mathcal{G}$ is covariant and $\mathcal{G} \circ \mathcal{F}=\mathrm{id}_{\mathscr{C}}$ and $\mathcal{F} \circ \mathcal{G}=\operatorname{id}_{\mathscr{D}}$. Consider $\mathcal{F}_{1}$ being a functor from $\mathscr{C}$ to $\mathscr{B}$ such that $\mathcal{F}_{1}$ is covariant and for every functor $\mathcal{G}$ from $\mathscr{C}$ to $\mathscr{B}$ such that $\mathcal{G}$ is covariant 
holds $\mathcal{F}_{1}=\mathcal{G}$. Set $\mathcal{F}_{2}=\mathcal{F}_{1} \circ \mathcal{G}$. For every functor $\mathcal{G}_{1}$ from $\mathscr{D}$ to $\mathscr{B}$ such that $\mathcal{G}_{1}$ is covariant holds $\mathcal{F}_{2}=\mathcal{G}_{1}$ by [15, (35)], [16, (10), (11)].

Let us note that every category which is empty is also initial.

Let $\mathscr{C}$ be a category. The functor $\mathbf{0} \rightarrow \mathscr{C}$ yielding a covariant functor from 0 to $\mathscr{C}$ is defined by

(Def. 9) not contradiction.

Now we state the proposition:

(38) Let us consider categories $\mathscr{C}, \mathscr{C}_{1}, \mathscr{C}_{2}$, a functor $\mathcal{F}_{1}$ from $\mathscr{C}_{1}$ to $\mathscr{C}$, and a functor $\mathcal{F}_{2}$ from $\mathscr{C}_{2}$ to $\mathscr{C}$. Suppose $\mathcal{F}_{1}$ is covariant and $\mathcal{F}_{2}$ is covariant. Then $\mathcal{F}_{1} \circ \mathbf{0} \rightarrow \mathscr{C}_{1}=\mathcal{F}_{2} \circ \mathbf{0} \rightarrow \mathscr{C}_{2}$.

\section{Categorical Products}

Let $\mathscr{C}$ be a category, $a, b, c$ be objects of $\mathscr{C}$, and $p_{1}$ be a morphism from $c$ to $a$. Assume $\operatorname{hom}(c, a) \neq \emptyset$. Let $p_{2}$ be a morphism from $c$ to $b$. Assume $\operatorname{hom}(c, b) \neq \emptyset$. We say that $\left\langle c, p_{1}, p_{2}\right\rangle$ is a product of $a$ and $b$ if and only if

(Def. 10) for every object $c_{1}$ of $\mathscr{C}$ and for every morphism $q_{1}$ from $c_{1}$ to $a$ and for every morphism $q_{2}$ from $c_{1}$ to $b$ such that $\operatorname{hom}\left(c_{1}, a\right) \neq \emptyset$ and $\operatorname{hom}\left(c_{1}, b\right) \neq$ $\emptyset$ holds $\operatorname{hom}\left(c_{1}, c\right) \neq \emptyset$ and there exists a morphism $h$ from $c_{1}$ to $c$ such that $p_{1} \cdot h=q_{1}$ and $p_{2} \cdot h=q_{2}$ and for every morphism $h_{1}$ from $c_{1}$ to $c$ such that $p_{1} \cdot h_{1}=q_{1}$ and $p_{2} \cdot h_{1}=q_{2}$ holds $h=h_{1}$.

Now we state the propositions:

(39) Let us consider a category $\mathscr{C}$, objects $c_{1}, c_{2}, a, b$ of $\mathscr{C}$, a morphism $p_{1}$ from $a$ to $c_{1}$, a morphism $p_{2}$ from $a$ to $c_{2}$, a morphism $q_{1}$ from $b$ to $c_{1}$, and a morphism $q_{2}$ from $b$ to $c_{2}$. Suppose $\operatorname{hom}\left(a, c_{1}\right) \neq \emptyset$ and $\operatorname{hom}\left(a, c_{2}\right) \neq \emptyset$ and $\operatorname{hom}\left(b, c_{1}\right) \neq \emptyset$ and $\operatorname{hom}\left(b, c_{2}\right) \neq \emptyset$ and $\left\langle a, p_{1}, p_{2}\right\rangle$ is a product of $c_{1}$ and $c_{2}$ and $\left\langle b, q_{1}, q_{2}\right\rangle$ is a product of $c_{1}$ and $c_{2}$. Then $a$ and $b$ are isomorphic.

ProOF: There exists a morphism $f$ from $a$ to $b$ and there exists a morphism $g$ from $b$ to $a$ such that $\operatorname{hom}(a, b) \neq \emptyset$ and $\operatorname{hom}(b, a) \neq \emptyset$ and $g \cdot f=\mathrm{id}-a$ and $f \cdot g=$ id- $b$ by [16, (23), (18)].

(40) Let us consider a category $\mathscr{C}$, objects $c_{1}, c_{2}, d$ of $\mathscr{C}$, a morphism $p_{1}$ from $d$ to $c_{1}$, and a morphism $p_{2}$ from $d$ to $c_{2}$. Suppose $\operatorname{hom}\left(d, c_{1}\right) \neq \emptyset$ and $\operatorname{hom}\left(d, c_{2}\right) \neq \emptyset$ and $\left\langle d, p_{1}, p_{2}\right\rangle$ is a product of $c_{1}$ and $c_{2}$. Then $\left\langle d, p_{2}, p_{1}\right\rangle$ is a product of $c_{2}$ and $c_{1}$.

Let $\mathscr{C}$ be a category. We say that $\mathscr{C}$ has binary products if and only if

(Def. 11) for every objects $a, b$ of $\mathscr{C}$, there exists an object $d$ of $\mathscr{C}$ and there exists a morphism $p_{1}$ from $d$ to $a$ and there exists a morphism $p_{2}$ from $d$ to $b$ 
such that $\operatorname{hom}(d, a) \neq \emptyset$ and $\operatorname{hom}(d, b) \neq \emptyset$ and $\left\langle d, p_{1}, p_{2}\right\rangle$ is a product of $a$ and $b$.

Now we state the proposition:

(41) 1 has binary products.

Proof: Set $\mathscr{C}=1$. Consider $f$ being a morphism of 1 such that $f$ is identity and $\mathrm{Ob} \mathbf{1}=\{f\}$ and Mor $\mathbf{1}=\{f\}$. For every objects $o_{1}, o_{2}$ of $\mathscr{C}$, every morphism of $\mathscr{C}$ is a morphism from $o_{1}$ to $o_{2}$ by [16, (20)]. Reconsider $p_{1}=f$ as a morphism from $a$ to $a$. Reconsider $p_{2}=f$ as a morphism from $a$ to $b$. For every object $c_{1}$ of $\mathscr{C}$ and for every morphism $q_{1}$ from $c_{1}$ to $a$ and for every morphism $q_{2}$ from $c_{1}$ to $b$ such that hom $\left(c_{1}, a\right) \neq \emptyset$ and $\operatorname{hom}\left(c_{1}, b\right) \neq \emptyset$ holds $\operatorname{hom}\left(c_{1}, a\right) \neq \emptyset$ and there exists a morphism $h$ from $c_{1}$ to $a$ such that $p_{1} \cdot h=q_{1}$ and $p_{2} \cdot h=q_{2}$ and for every morphism $h_{1}$ from $c_{1}$ to $a$ such that $p_{1} \cdot h_{1}=q_{1}$ and $p_{2} \cdot h_{1}=q_{2}$ holds $h=h_{1}$.

Observe that there exists a category which has binary products.

Let $\mathscr{C}$ be a category with binary products and $c_{1}, c_{2}$ be objects of $\mathscr{C}$.

A categorical product of $c_{1}$ and $c_{2}$ is a triple object and is defined by

(Def. 12) there exists an object $d$ of $\mathscr{C}$ and there exists a morphism $p_{1}$ from $d$ to $c_{1}$ and there exists a morphism $p_{2}$ from $d$ to $c_{2}$ such that $i t=\left\langle d, p_{1}, p_{2}\right\rangle$ and $\operatorname{hom}\left(d, c_{1}\right) \neq \emptyset$ and $\operatorname{hom}\left(d, c_{2}\right) \neq \emptyset$ and $\left\langle d, p_{1}, p_{2}\right\rangle$ is a product of $c_{1}$ and $c_{2}$.

The functor $c_{1} \times c_{2}$ yielding an object of $\mathscr{C}$ is defined by the term

(Def. 13) (the categorical product of $c_{1}$ and $\left.c_{2}\right)_{1,3}$.

The functor $\pi_{1}\left(c_{1} \otimes c_{2}\right)$ yielding a morphism from $c_{1} \times c_{2}$ to $c_{1}$ is defined by the term

(Def. 14) (the categorical product of $c_{1}$ and $\left.c_{2}\right)_{2,3}$.

The functor $\pi_{2}\left(c_{1} \otimes c_{2}\right)$ yielding a morphism from $c_{1} \times c_{2}$ to $c_{2}$ is defined by the term

(Def. 15) (the categorical product of $c_{1}$ and $\left.c_{2}\right)_{3,3}$.

Now we state the propositions:

(42) Let us consider a category $\mathscr{C}$ with binary products, and objects $a, b$ of $\mathscr{C}$. Then

(i) $\left\langle a \times b, \pi_{1}(a \otimes b), \pi_{2}(a \otimes b)\right\rangle$ is a product of $a$ and $b$, and

(ii) $\operatorname{hom}(a \times b, a) \neq \emptyset$, and

(iii) $\operatorname{hom}(a \times b, b) \neq \emptyset$.

(43) Let us consider a category $\mathscr{C}$ with binary products, and objects $a, b, c$ of $\mathscr{C}$. Suppose $\operatorname{hom}(c, a) \neq \emptyset$ and $\operatorname{hom}(c, b) \neq \emptyset$. Then $\operatorname{hom}(c, a \times b) \neq \emptyset$. The theorem is a consequence of (42). 
(44) Let us consider a category $\mathscr{C}$ with binary products, and objects $a, b, c, d$ of $\mathscr{C}$. Suppose $\operatorname{hom}(a, b) \neq \emptyset$ and $\operatorname{hom}(c, d) \neq \emptyset$. Then $\operatorname{hom}(a \times c, b \times d) \neq \emptyset$. The theorem is a consequence of (42).

Let $\mathscr{C}$ be a category with binary products, $a, b, c, d$ be objects of $\mathscr{C}$, and $f$ be a morphism from $a$ to $b$. Assume $\operatorname{hom}(a, b) \neq \emptyset$. Let $g$ be a morphism from $c$ to $d$. Assume $\operatorname{hom}(c, d) \neq \emptyset$. The functor $f \times g$ yielding a morphism from $a \times c$ to $b \times d$ is defined by

(Def. 16) $f \cdot \pi_{1}(a \otimes c)=\pi_{1}(b \otimes d) \cdot i t$ and $g \cdot \pi_{2}(a \otimes c)=\pi_{2}(b \otimes d) \cdot i t$.

Let $\mathscr{C}_{1}, \mathscr{C}_{2}, \mathscr{D}$ be categories and $\mathcal{P}_{1}$ be a functor from $\mathscr{D}$ to $\mathscr{C}_{1}$. Assume $\mathcal{P}_{1}$ is covariant. Let $\mathcal{P}_{2}$ be a functor from $\mathscr{D}$ to $\mathscr{C}_{2}$. Assume $\mathcal{P}_{2}$ is covariant. We say that $\left\langle\mathscr{D}, \mathcal{P}_{1}, \mathcal{P}_{2}\right\rangle$ is a product of $\mathscr{C}_{1}$ and $\mathscr{C}_{2}$ if and only if

(Def. 17) for every category $\mathscr{D}_{1}$ and for every functor $\mathcal{G}_{1}$ from $\mathscr{D}_{1}$ to $\mathscr{C}_{1}$ and for every functor $\mathcal{G}_{2}$ from $\mathscr{D}_{1}$ to $\mathscr{C}_{2}$ such that $\mathcal{G}_{1}$ is covariant and $\mathcal{G}_{2}$ is covariant there exists a functor $\mathcal{H}$ from $\mathscr{D}_{1}$ to $\mathscr{D}$ such that $\mathcal{H}$ is covariant and $\mathcal{P}_{1} \circ \mathcal{H}=$ $\mathcal{G}_{1}$ and $\mathcal{P}_{2} \circ \mathcal{H}=\mathcal{G}_{2}$ and for every functor $\mathcal{H}_{1}$ from $\mathscr{D}_{1}$ to $\mathscr{D}$ such that $\mathcal{H}_{1}$ is covariant and $\mathcal{P}_{1} \circ \mathcal{H}_{1}=\mathcal{G}_{1}$ and $\mathcal{P}_{2} \circ \mathcal{H}_{1}=\mathcal{G}_{2}$ holds $\mathcal{H}=\mathcal{H}_{1}$.

Now we state the propositions:

(45) Let us consider categories $\mathscr{C}_{1}, \mathscr{C}_{2}, \mathscr{A}, \mathscr{B}$, a functor $\mathcal{P}_{1}$ from $\mathscr{A}$ to $\mathscr{C}_{1}$, a functor $\mathcal{P}_{2}$ from $\mathscr{A}$ to $\mathscr{C}_{2}$, a functor $\mathcal{Q}_{1}$ from $\mathscr{B}$ to $\mathscr{C}_{1}$, and a functor $\mathcal{Q}_{2}$ from $\mathscr{B}$ to $\mathscr{C}_{2}$. Suppose $\mathcal{P}_{1}$ is covariant and $\mathcal{P}_{2}$ is covariant and $\mathcal{Q}_{1}$ is covariant and $\mathcal{Q}_{2}$ is covariant and $\left\langle\mathscr{A}, \mathcal{P}_{1}, \mathcal{P}_{2}\right\rangle$ is a product of $\mathscr{C}_{1}$ and $\mathscr{C}_{2}$ and $\left\langle\mathscr{B}, \mathcal{Q}_{1}, \mathcal{Q}_{2}\right\rangle$ is a product of $\mathscr{C}_{1}$ and $\mathscr{C}_{2}$. Then $\mathscr{A} \cong \mathscr{B}$.

Proof: There exists a functor $\mathcal{F}_{4}$ from $\mathscr{A}$ to $\mathscr{B}$ and there exists a functor $\mathcal{G}_{3}$ from $\mathscr{B}$ to $\mathscr{A}$ such that $\mathcal{F}_{4}$ is covariant and $\mathcal{G}_{3}$ is covariant and $\mathcal{G}_{3} \circ \mathcal{F}_{4}=$ $\operatorname{id}_{\mathscr{A}}$ and $\mathcal{F}_{4} \circ \mathcal{G}_{3}=\mathrm{id}_{\mathscr{B}}$ by [16, (10), (11)], [15, (35)].

(46) Let us consider categories $\mathscr{C}_{1}, \mathscr{C}_{2}, \mathscr{D}$, a functor $\mathcal{P}_{1}$ from $\mathscr{D}$ to $\mathscr{C}_{1}$, and a functor $\mathcal{P}_{2}$ from $\mathscr{D}$ to $\mathscr{C}_{2}$. Suppose $\mathcal{P}_{1}$ is covariant and $\mathcal{P}_{2}$ is covariant and $\left\langle\mathscr{D}, \mathcal{P}_{1}, \mathcal{P}_{2}\right\rangle$ is a product of $\mathscr{C}_{1}$ and $\mathscr{C}_{2}$. Then $\left\langle\mathscr{D}, \mathcal{P}_{2}, \mathcal{P}_{1}\right\rangle$ is a product of $\mathscr{C}_{2}$ and $\mathscr{C}_{1}$.

Let $\mathscr{C}, \mathscr{C}_{1}, \mathscr{C}_{2}$ be categories, $\mathcal{F}_{1}$ be a functor from $\mathscr{C}_{1}$ to $\mathscr{C}$, and $\mathcal{F}_{2}$ be a functor from $\mathscr{C}_{2}$ to $\mathscr{C}$. We introduce the notation $\mathcal{F}_{1} \otimes \mathcal{F}_{2}$ as a synonym of $\llbracket \mathcal{F}_{1}, \mathcal{F}_{2} \rrbracket$.

Now we state the proposition:

(47) Let us consider categories $\mathscr{C}_{1}, \mathscr{C}_{2}$. Then $\left\langle\mathscr{C}_{1} \rightarrow \mathbf{1} \otimes \mathscr{C}_{2} \rightarrow \mathbf{1}, \pi_{1}\left(\left(\mathscr{C}_{1} \rightarrow \mathbf{1}\right) \otimes\right.\right.$ $\left.\left.\left(\mathscr{C}_{2} \rightarrow \mathbf{1}\right)\right), \pi_{2}\left(\left(\mathscr{C}_{1} \rightarrow \mathbf{1}\right) \otimes\left(\mathscr{C}_{2} \rightarrow \mathbf{1}\right)\right)\right\rangle$ is a product of $\mathscr{C}_{1}$ and $\mathscr{C}_{2}$.

Proof: Set $\mathcal{F}_{1}=\mathscr{C}_{1} \rightarrow \mathbf{1}$. Set $\mathcal{F}_{2}=\mathscr{C}_{2} \rightarrow \mathbf{1}$. For every category $\mathscr{D}_{1}$ and for every functor $\mathcal{G}_{1}$ from $\mathscr{D}_{1}$ to $\mathscr{C}_{1}$ and for every functor $\mathcal{G}_{2}$ from $\mathscr{D}_{1}$ to $\mathscr{C}_{2}$ such that $\mathcal{G}_{1}$ is covariant and $\mathcal{G}_{2}$ is covariant there exists a functor $\mathcal{H}$ from $\mathscr{D}_{1}$ to $\mathcal{F}_{1} \otimes \mathcal{F}_{2}$ such that $\mathcal{H}$ is covariant and $\pi_{1}\left(\mathcal{F}_{1} \otimes \mathcal{F}_{2}\right) \circ \mathcal{H}=\mathcal{G}_{1}$ and 
$\pi_{2}\left(\mathcal{F}_{1} \otimes \mathcal{F}_{2}\right) \circ \mathcal{H}=\mathcal{G}_{2}$ and for every functor $\mathcal{H}_{1}$ from $\mathscr{D}_{1}$ to $\mathcal{F}_{1} \otimes \mathcal{F}_{2}$ such that $\mathcal{H}_{1}$ is covariant and $\pi_{1}\left(\mathcal{F}_{1} \otimes \mathcal{F}_{2}\right) \circ \mathcal{H}_{1}=\mathcal{G}_{1}$ and $\pi_{2}\left(\mathcal{F}_{1} \otimes \mathcal{F}_{2}\right) \circ \mathcal{H}_{1}=\mathcal{G}_{2}$ holds $\mathcal{H}=\mathcal{H}_{1}$ by [16, (52)], (29).

Let $\mathscr{C}_{1}, \mathscr{C}_{2}$ be categories.

A categorical product of $\mathscr{C}_{1}$ and $\mathscr{C}_{2}$ is a triple object and is defined by

(Def. 18) there exists a strict category $\mathscr{D}$ and there exists a functor $\mathcal{P}_{1}$ from $\mathscr{D}$ to $\mathscr{C}_{1}$ and there exists a functor $\mathcal{P}_{2}$ from $\mathscr{D}$ to $\mathscr{C}_{2}$ such that $i t=\left\langle\mathscr{D}, \mathcal{P}_{1}, \mathcal{P}_{2}\right\rangle$ and $\mathcal{P}_{1}$ is covariant and $\mathcal{P}_{2}$ is covariant and $\left\langle\mathscr{D}, \mathcal{P}_{1}, \mathcal{P}_{2}\right\rangle$ is a product of $\mathscr{C}_{1}$ and $\mathscr{C}_{2}$.

The functor $\mathscr{C}_{1} \times \mathscr{C}_{2}$ yielding a strict category is defined by the term

(Def. 19) (the categorical product of $\mathscr{C}_{1}$ and $\left.\mathscr{C}_{2}\right)_{\mathbf{1}, 3}$.

The functor $\pi_{1}\left(\mathscr{C}_{1} \otimes \mathscr{C}_{2}\right)$ yielding a functor from $\mathscr{C}_{1} \times \mathscr{C}_{2}$ to $\mathscr{C}_{1}$ is defined by the term

(Def. 20) (the categorical product of $\mathscr{C}_{1}$ and $\left.\mathscr{C}_{2}\right)_{\mathbf{2}, 3}$.

The functor $\pi_{2}\left(\mathscr{C}_{1} \otimes \mathscr{C}_{2}\right)$ yielding a functor from $\mathscr{C}_{1} \times \mathscr{C}_{2}$ to $\mathscr{C}_{2}$ is defined by the term

(Def. 21) (the categorical product of $\mathscr{C}_{1}$ and $\left.\mathscr{C}_{2}\right)_{\mathbf{3}, 3}$.

Now we state the proposition:

(48) Let us consider categories $\mathscr{C}_{1}, \mathscr{C}_{2}$. Then $\left\langle\mathscr{C}_{1} \times \mathscr{C}_{2}, \pi_{1}\left(\mathscr{C}_{1} \nabla \mathscr{C}_{2}\right), \pi_{2}\left(\mathscr{C}_{1} \nabla \mathscr{C}_{2}\right)\right\rangle$ is a product of $\mathscr{C}_{1}$ and $\mathscr{C}_{2}$.

Let $\mathscr{C}_{1}, \mathscr{C}_{2}$ be categories. Note that $\pi_{1}\left(\mathscr{C}_{1} \otimes \mathscr{C}_{2}\right)$ is covariant and $\pi_{2}\left(\mathscr{C}_{1} \otimes \mathscr{C}_{2}\right)$ is covariant.

Now we state the proposition:

(49) Let us consider categories $\mathscr{C}_{1}, \mathscr{C}_{2}$. Then $\mathscr{C}_{1} \times \mathscr{C}_{2}$ is not empty if and only if $\mathscr{C}_{1}$ is not empty and $\mathscr{C}_{2}$ is not empty. The theorem is a consequence of (48).

Let $\mathscr{C}_{1}$ be an empty category and $\mathscr{C}_{2}$ be a category. One can verify that $\mathscr{C}_{1} \times \mathscr{C}_{2}$ is empty.

Let $\mathscr{C}_{1}$ be a category and $\mathscr{C}_{2}$ be an empty category. Observe that $\mathscr{C}_{1} \times \mathscr{C}_{2}$ is empty.

Let $\mathscr{C}_{1}$ be a non empty category and $\mathscr{C}_{2}$ be a non empty category. One can verify that $\mathscr{C}_{1} \times \mathscr{C}_{2}$ is non empty.

Let $\mathscr{C}_{1}, \mathscr{C}_{2}, \mathscr{D}_{1}, \mathscr{D}_{2}$ be categories, $\mathcal{F}_{1}$ be a functor from $\mathscr{C}_{1}$ to $\mathscr{D}_{1}$, and $\mathcal{F}_{2}$ be a functor from $\mathscr{C}_{2}$ to $\mathscr{D}_{2}$. Assume $\mathcal{F}_{1}$ is covariant and $\mathcal{F}_{2}$ is covariant. The functor $\mathcal{F}_{1} \times \mathcal{F}_{2}$ yielding a functor from $\mathscr{C}_{1} \times \mathscr{C}_{2}$ to $\mathscr{D}_{1} \times \mathscr{D}_{2}$ is defined by

(Def. 22) it is covariant and $\mathcal{F}_{1} \circ \pi_{1}\left(\mathscr{C}_{1} \otimes \mathscr{C}_{2}\right)=\pi_{1}\left(\mathscr{D}_{1} \otimes \mathscr{D}_{2}\right) \circ i t$ and $\mathcal{F}_{2} \circ \pi_{2}\left(\mathscr{C}_{1} \otimes \mathscr{C}_{2}\right)=$ $\pi_{2}\left(\mathscr{D}_{1} \otimes \mathscr{D}_{2}\right) \circ i t$.

Now we state the propositions: 
(50) Let us consider categories $\mathscr{A}_{1}, \mathscr{A}_{2}, \mathscr{B}_{1}, \mathscr{B}_{2}, \mathscr{C}_{1}, \mathscr{C}_{2}$, a functor $\mathcal{F}_{1}$ from $\mathscr{A}_{1}$ to $\mathscr{B}_{1}$, a functor $\mathcal{F}_{2}$ from $\mathscr{A}_{2}$ to $\mathscr{B}_{2}$, a functor $\mathcal{G}_{1}$ from $\mathscr{B}_{1}$ to $\mathscr{C}_{1}$, and a functor $\mathcal{G}_{2}$ from $\mathscr{B}_{2}$ to $\mathscr{C}_{2}$. Suppose $\mathcal{F}_{1}$ is covariant and $\mathcal{G}_{1}$ is covariant and $\mathcal{F}_{2}$ is covariant and $\mathcal{G}_{2}$ is covariant. Then $\left(\mathcal{G}_{1} \times \mathcal{G}_{2}\right) \circ\left(\mathcal{F}_{1} \times \mathcal{F}_{2}\right)=$ $\left(\mathcal{G}_{1} \circ \mathcal{F}_{1}\right) \times\left(\mathcal{G}_{2} \circ \mathcal{F}_{2}\right)$.

(51) Let us consider categories $\mathscr{C}_{1}, \mathscr{C}_{2}$. Then $\operatorname{id}_{\mathscr{C}_{1}} \times \mathrm{id}_{\mathscr{C}_{2}}=\mathrm{id}_{\mathscr{C}_{1} \times \mathscr{C}_{2}}$.

Let $x, y$ be objects. We introduce the notation $\operatorname{KuratowskiPair}(x, y)$ as a synonym of $\langle x, y\rangle$.

Let $\mathscr{C}_{1}, \mathscr{C}_{2}$ be categories, $f_{1}$ be a morphism of $\mathscr{C}_{1}$, and $f_{2}$ be a morphism of $\mathscr{C}_{2}$. The functor $\left\langle f_{1}, f_{2}\right\rangle$ yielding a morphism of $\mathscr{C}_{1} \times \mathscr{C}_{2}$ is defined by

(Def. 23) (i) $\pi_{1}\left(\mathscr{C}_{1} \otimes \mathscr{C}_{2}\right)(i t)=f_{1}$ and $\pi_{2}\left(\mathscr{C}_{1} \otimes \mathscr{C}_{2}\right)(i t)=f_{2}$, if $\mathscr{C}_{1}$ is not empty and $\mathscr{C}_{2}$ is not empty,

(ii) $i t=$ the morphism of $\mathscr{C}_{1} \times \mathscr{C}_{2}$, otherwise.

Now we state the propositions:

(52) Let us consider categories $\mathscr{C}_{1}, \mathscr{C}_{2}$, and a morphism $f$ of $\mathscr{C}_{1} \times \mathscr{C}_{2}$. Then there exists a morphism $f_{1}$ of $\mathscr{C}_{1}$ and there exists a morphism $f_{2}$ of $\mathscr{C}_{2}$ such that $f=\left\langle f_{1}, f_{2}\right\rangle$.

(53) Let us consider non empty categories $\mathscr{C}_{1}, \mathscr{C}_{2}$, morphisms $f_{1}, g_{1}$ of $\mathscr{C}_{1}$, and morphisms $f_{2}, g_{2}$ of $\mathscr{C}_{2}$. Suppose $\left\langle f_{1}, f_{2}\right\rangle=\left\langle g_{1}, g_{2}\right\rangle$. Then

(i) $f_{1}=g_{1}$, and

(ii) $f_{2}=g_{2}$.

Let us consider categories $\mathscr{C}_{1}, \mathscr{C}_{2}$, morphisms $f_{1}, g_{1}$ of $\mathscr{C}_{1}$, and morphisms $f_{2}, g_{2}$ of $\mathscr{C}_{2}$. Now we state the propositions:

(54) $\left\langle f_{1}, f_{2}\right\rangle \triangleright\left\langle g_{1}, g_{2}\right\rangle$ if and only if $f_{1} \triangleright g_{1}$ and $f_{2} \triangleright g_{2}$.

(55) Suppose $f_{1} \triangleright g_{1}$ and $f_{2} \triangleright g_{2}$. Then $\left\langle f_{1}, f_{2}\right\rangle \circ\left\langle g_{1}, g_{2}\right\rangle=\left\langle f_{1} \circ g_{1}, f_{2} \circ g_{2}\right\rangle$. The theorem is a consequence of (54) and (13).

Now we state the propositions:

(56) Let us consider categories $\mathscr{C}_{1}, \mathscr{C}_{2}$, a morphism $f_{1}$ of $\mathscr{C}_{1}$, a morphism $f_{2}$ of $\mathscr{C}_{2}$, and a morphism $f$ of $\mathscr{C}_{1} \times \mathscr{C}_{2}$. Suppose $f=\left\langle f_{1}, f_{2}\right\rangle$ and $\mathscr{C}_{1}$ is not empty and $\mathscr{C}_{2}$ is not empty. Then $f$ is identity if and only if $f_{1}$ is identity and $f_{2}$ is identity. The theorem is a consequence of (52), (54), (55), and (4).

(57) Let us consider non empty categories $\mathscr{C}_{1}, \mathscr{C}_{2}$, categories $\mathscr{D}_{1}, \mathscr{D}_{2}$, a functor $\mathcal{F}_{1}$ from $\mathscr{C}_{1}$ to $\mathscr{D}_{1}$, a functor $\mathcal{F}_{2}$ from $\mathscr{C}_{2}$ to $\mathscr{D}_{2}$, a morphism $c_{1}$ of $\mathscr{C}_{1}$, and a morphism $c_{2}$ of $\mathscr{C}_{2}$. Suppose $\mathcal{F}_{1}$ is covariant and $\mathcal{F}_{2}$ is covariant. Then $\left(\mathcal{F}_{1} \times \mathcal{F}_{2}\right)\left(\left\langle c_{1}, c_{2}\right\rangle\right)=\left\langle\mathcal{F}_{1}\left(c_{1}\right), \mathcal{F}_{2}\left(c_{2}\right)\right\rangle$. 


\section{Natural Transformations}

Let $\mathscr{C}_{1}, \mathscr{C}_{2}$ be categories, $\mathcal{F}_{1}, \mathcal{F}_{2}$ be functors from $\mathscr{C}_{1}$ to $\mathscr{C}_{2}$, and $\tau$ be a functor from $\mathscr{C}_{1}$ to $\mathscr{C}_{2}$. We say that $\tau$ is a natural transformation of $\mathcal{F}_{1}$ and $\mathcal{F}_{2}$ if and only if

(Def. 24) for every morphisms $f_{1}, f_{2}$ of $\mathscr{C}_{1}$ such that $f_{1} \triangleright f_{2}$ holds $\tau\left(f_{1}\right) \triangleright \mathcal{F}_{1}\left(f_{2}\right)$ and $\mathcal{F}_{2}\left(f_{1}\right) \triangleright \tau\left(f_{2}\right)$ and $\tau\left(f_{1} \circ f_{2}\right)=\tau\left(f_{1}\right) \circ \mathcal{F}_{1}\left(f_{2}\right)$ and $\tau\left(f_{1} \circ f_{2}\right)=\mathcal{F}_{2}\left(f_{1}\right) \circ \tau\left(f_{2}\right)$.

Now we state the propositions:

(58) Let us consider categories $\mathscr{C}_{1}, \mathscr{C}_{2}$, functors $\mathcal{F}_{1}, \mathcal{F}_{2}$ from $\mathscr{C}_{1}$ to $\mathscr{C}_{2}$, and a functor $\tau$ from $\mathscr{C}_{1}$ to $\mathscr{C}_{2}$. Suppose $\mathcal{F}_{1}$ is covariant and $\mathcal{F}_{2}$ is covariant. Then $\tau$ is a natural transformation of $\mathcal{F}_{1}$ and $\mathcal{F}_{2}$ if and only if for every morphisms $f, f_{1}, f_{2}$ of $\mathscr{C}_{1}$ such that $f_{1}$ is identity and $f_{2}$ is identity and $f_{1} \triangleright f$ and $f \triangleright f_{2}$ holds $\tau\left(f_{1}\right) \triangleright \mathcal{F}_{1}(f)$ and $\mathcal{F}_{2}(f) \triangleright \tau\left(f_{2}\right)$ and $\tau(f)=\tau\left(f_{1}\right) \circ$ $\mathcal{F}_{1}(f)$ and $\tau(f)=\mathcal{F}_{2}(f) \circ \tau\left(f_{2}\right)$.

PROOF: For every morphisms $g_{1}, g_{2}$ of $\mathscr{C}_{1}$ such that $g_{1} \triangleright g_{2}$ holds $\tau\left(g_{1}\right) \triangleright$ $\mathcal{F}_{1}\left(g_{2}\right)$ and $\mathcal{F}_{2}\left(g_{1}\right) \triangleright \tau\left(g_{2}\right)$ and $\tau\left(g_{1} \circ g_{2}\right)=\tau\left(g_{1}\right) \circ \mathcal{F}_{1}\left(g_{2}\right)$ and $\tau\left(g_{1} \circ g_{2}\right)=$ $\mathcal{F}_{2}\left(g_{1}\right) \circ \tau\left(g_{2}\right)$ by [15, (1)], (5), (3), (13).

(59) Let us consider non empty categories $\mathscr{C}_{1}, \mathscr{C}_{2}$, covariant functors $\mathcal{F}_{1}, \mathcal{F}_{2}$ from $\mathscr{C}_{1}$ to $\mathscr{C}_{2}$, and a function $\tau$ from $\mathrm{Ob} \mathscr{C}_{1}$ into Mor $\mathscr{C}_{2}$. Then there exists a functor $\tau_{1}$ from $\mathscr{C}_{1}$ to $\mathscr{C}_{2}$ such that $\left.\tau=\tau_{1}\right\rceil \mathrm{Ob} \mathscr{C}_{1}$ and $\tau_{1}$ is a natural transformation of $\mathcal{F}_{1}$ and $\mathcal{F}_{2}$ if and only if for every object $a$ of $\mathscr{C}_{1}, \tau(a) \in \operatorname{hom}\left(\mathcal{F}_{1}(a), \mathcal{F}_{2}(a)\right)$ and for every objects $a_{1}, a_{2}$ of $\mathscr{C}_{1}$ and for every morphism $f$ from $a_{1}$ to $a_{2}$ such that $\operatorname{hom}\left(a_{1}, a_{2}\right) \neq \emptyset$ holds $\tau\left(a_{2}\right) \circ \mathcal{F}_{1}(f)=\mathcal{F}_{2}(f) \circ \tau\left(a_{1}\right)$.

Proof: Define $\mathcal{P}$ [object, object] $\equiv$ for every morphism $f$ of $\mathscr{C}_{1}$ such that $\$_{1}=f$ holds $\$_{2}=\tau(\operatorname{cod} f) \circ \mathcal{F}_{1}(f)$. For every object $x$ such that $x \in$ the carrier of $\mathscr{C}_{1}$ there exists an object $y$ such that $y \in$ the carrier of $\mathscr{C}_{2}$ and $\mathcal{P}[x, y]$. Consider $\tau_{1}$ being a function from the carrier of $\mathscr{C}_{1}$ into the carrier of $\mathscr{C}_{2}$ such that for every object $x$ such that $x \in$ the carrier of $\mathscr{C}_{1}$ holds $\mathcal{P}\left[x, \tau_{1}(x)\right]$ from [7, Sch. 1]. For every object $x$ such that $x \in \operatorname{dom} \tau$ holds $\tau(x)=\left(\tau_{1}\left\lceil\mathrm{Ob} \mathscr{C}_{1}\right)(x)\right.$ by [15, (22)], [16, (20)], [15, (32)], [16, (5), (6)]. For every morphisms $f, f_{1}, f_{2}$ of $\mathscr{C}_{1}$ such that $f_{1}$ is identity and $f_{2}$ is identity and $f_{1} \triangleright f$ and $f \triangleright f_{2}$ holds $\tau_{1}\left(f_{1}\right) \triangleright \mathcal{F}_{1}(f)$ and $\mathcal{F}_{2}(f) \triangleright \tau_{1}\left(f_{2}\right)$ and $\tau_{1}(f)=\tau_{1}\left(f_{1}\right) \circ \mathcal{F}_{1}(f)$ and $\tau_{1}(f)=\mathcal{F}_{2}(f) \circ \tau_{1}\left(f_{2}\right)$ by [15, (22)], [16, (20), (6)], [15, (32)].

(60) Let us consider object-categories $\mathscr{C}, \mathscr{D}$, functors $\mathcal{F}_{1}, \mathcal{F}_{2}$ from $\mathscr{C}$ to $\mathscr{D}$, and functors $\mathcal{G}_{1}, \mathcal{G}_{2}, \tau$ from alter $\mathscr{C}$ to alter $\mathscr{D}$. Suppose $\mathcal{F}_{1}=\mathcal{G}_{1}$ and $\mathcal{F}_{2}=\mathcal{G}_{2}$ and $\tau$ is a natural transformation of $\mathcal{G}_{1}$ and $\mathcal{G}_{2}$. Then $(\operatorname{IdMap} \mathscr{C}) \cdot \tau$ is a natural transformation from $\mathcal{F}_{1}$ to $\mathcal{F}_{2}$. 
Proof: For every object $a$ of $\mathscr{C}, \tau\left(\operatorname{id}_{a}\right) \in \operatorname{hom}\left(\mathcal{F}_{1}(a), \mathcal{F}_{2}(a)\right)$ by [15, (41), (24), (42)]. Reconsider $\tau_{1}=\tau$ as a function from the carrier' of $\mathscr{C}$ into the carrier' of $\mathscr{D}$. There exists a transformation $t$ from $\mathcal{F}_{1}$ to $\mathcal{F}_{2}$ such that $t=(\operatorname{IdMap} \mathscr{C}) \cdot \tau_{1}$ and for every objects $a, b$ of $\mathscr{C}$ such that $\operatorname{hom}(a, b) \neq \emptyset$ for every morphism $f$ from $a$ to $b, t(b) \cdot \mathcal{F}_{1 f}=\mathcal{F}_{2 f} \cdot t(a)$ by [6, (13)], [5, (1), (15), (21)]. Consider $t$ being a transformation from $\mathcal{F}_{1}$ to $\mathcal{F}_{2}$ such that $t=(\operatorname{IdMap} \mathscr{C}) \cdot \tau_{1}$ and for every objects $a, b$ of $\mathscr{C}$ such that hom $(a, b) \neq \emptyset$ for every morphism $f$ from $a$ to $b, t(b) \cdot \mathcal{F}_{1 f}=\mathcal{F}_{2 f} \cdot t(a)$.

Let $\mathscr{C}, \mathscr{D}$ be categories and $\mathcal{F}_{1}, \mathcal{F}_{2}$ be functors from $\mathscr{C}$ to $\mathscr{D}$. We say that $\mathcal{F}_{1}$ is naturally transformable to $\mathcal{F}_{2}$ if and only if

(Def. 25) there exists a functor $\tau$ from $\mathscr{C}$ to $\mathscr{D}$ such that $\tau$ is a natural transformation of $\mathcal{F}_{1}$ and $\mathcal{F}_{2}$.

Assume $\mathcal{F}_{1}$ is naturally transformable to $\mathcal{F}_{2}$.

A natural transformation from $\mathcal{F}_{1}$ to $\mathcal{F}_{2}$ is a functor from $\mathscr{C}$ to $\mathscr{D}$ and is defined by

(Def. 26) it is a natural transformation of $\mathcal{F}_{1}$ and $\mathcal{F}_{2}$.

Now we state the proposition:

(61) Let us consider categories $\mathscr{C}, \mathscr{D}$, and a functor $\mathcal{F}$ from $\mathscr{C}$ to $\mathscr{D}$. Suppose $\mathcal{F}$ is covariant. Then $\mathcal{F}$ is a natural transformation of $\mathcal{F}$ and $\mathcal{F}$. The theorem is a consequence of (58).

Let $\mathscr{C}, \mathscr{D}$ be categories and $\mathcal{F}, \mathcal{F}_{1}, \mathcal{F}_{2}$ be functors from $\mathscr{C}$ to $\mathscr{D}$. Assume $\mathcal{F}_{1}$ is naturally transformable to $\mathcal{F}$ and $\mathcal{F}$ is naturally transformable to $\mathcal{F}_{2}$ and $\mathcal{F}$ is covariant and $\mathcal{F}_{1}$ is covariant and $\mathcal{F}_{2}$ is covariant. Let $\tau_{1}$ be a natural transformation from $\mathcal{F}_{1}$ to $\mathcal{F}$ and $\tau_{2}$ be a natural transformation from $\mathcal{F}$ to $\mathcal{F}_{2}$. The functor $\tau_{2} \circ \tau_{1}$ yielding a natural transformation from $\mathcal{F}_{1}$ to $\mathcal{F}_{2}$ is defined by

(Def. 27) for every morphisms $f, f_{1}, f_{2}$ of $\mathscr{C}$ such that $f_{1}$ is identity and $f_{2}$ is identity and $f \triangleright f_{1}$ and $f_{2} \triangleright f$ holds $i t(f)=\left(\tau_{2}\left(f_{2}\right) \circ \mathcal{F}(f)\right) \circ \tau_{1}\left(f_{1}\right)$.

Now we state the proposition:

(62) Let us consider categories $\mathscr{C}, \mathscr{D}$, and functors $\mathcal{F}, \mathcal{F}_{1}, \mathcal{F}_{2}$ from $\mathscr{C}$ to $\mathscr{D}$. Suppose $\mathcal{F}_{1}$ is naturally transformable to $\mathcal{F}$ and $\mathcal{F}$ is naturally transformable to $\mathcal{F}_{2}$ and covariant and $\mathcal{F}_{1}$ is covariant and $\mathcal{F}_{2}$ is covariant. Then $\mathcal{F}_{1}$ is naturally transformable to $\mathcal{F}_{2}$.

Let $\mathscr{C}_{1}, \mathscr{C}_{2}$ be categories. The functor Functors $\left(\mathscr{C}_{2}, \mathscr{C}_{1}\right)$ yielding a strict category is defined by

(Def. 28) the carrier of it $=\left\{\left\langle\left\langle\mathcal{F}_{1}, \mathcal{F}_{2}\right\rangle, \tau\right\rangle\right.$, where $\mathcal{F}_{1}, \mathcal{F}_{2}$ are functors from $\mathscr{C}_{1}$ to $\mathscr{C}_{2}, \tau$ is a natural transformation from $\mathcal{F}_{1}$ to $\mathcal{F}_{2}: \mathcal{F}_{1}$ is covariant and $\mathcal{F}_{2}$ is covariant and $\mathcal{F}_{1}$ is naturally transformable to $\left.\mathcal{F}_{2}\right\}$ and the composi- 
tion of it $=\left\{\left\langle\left\langle x_{2}, x_{1}\right\rangle, x_{3}\right\rangle\right.$, where $x_{1}, x_{2}, x_{3}$ are elements of the carrier of it : there exist functors $\mathcal{F}_{1}, \mathcal{F}_{2}, \mathcal{F}_{3}$ from $\mathscr{C}_{1}$ to $\mathscr{C}_{2}$ and there exists a natural transformation $\tau_{1}$ from $\mathcal{F}_{1}$ to $\mathcal{F}_{2}$ and there exists a natural transformati-on $\tau_{2}$ from $\mathcal{F}_{2}$ to $\mathcal{F}_{3}$ such that $x_{1}=\left\langle\left\langle\mathcal{F}_{1}, \mathcal{F}_{2}\right\rangle, \tau_{1}\right\rangle$ and $x_{2}=$ $\left\langle\left\langle\mathcal{F}_{2}, \mathcal{F}_{3}\right\rangle, \tau_{2}\right\rangle$ and $\left.x_{3}=\left\langle\left\langle\mathcal{F}_{1}, \mathcal{F}_{3}\right\rangle, \tau_{2} \circ \tau_{1}\right\rangle\right\}$.

Let $\mathscr{C}_{1}$ be a non empty category and $\mathscr{C}_{2}$ be an empty category. One can verify that Functors $\left(\mathscr{C}_{2}, \mathscr{C}_{1}\right)$ is empty.

Let $\mathscr{C}_{1}$ be an empty category and $\mathscr{C}_{2}$ be a category. Let us observe that Functors $\left(\mathscr{C}_{2}, \mathscr{C}_{1}\right)$ is non empty and trivial.

Let $\mathscr{C}_{1}$ be a non empty category and $\mathscr{C}_{2}$ be a non empty category. Let us note that Functors $\left(\mathscr{C}_{2}, \mathscr{C}_{1}\right)$ is non empty.

Now we state the proposition:

(63) Let us consider non empty categories $\mathscr{C}_{1}, \mathscr{C}_{2}$, and morphisms $f_{1}, f_{2}$ of Functors $\left(\mathscr{C}_{2}, \mathscr{C}_{1}\right)$. Then $f_{1} \triangleright f_{2}$ if and only if there exist covariant functors $\mathcal{F}, \mathcal{F}_{1}, \mathcal{F}_{2}$ from $\mathscr{C}_{1}$ to $\mathscr{C}_{2}$ and there exists a natural transformation $\tau_{1}$ from $\mathcal{F}_{1}$ to $\mathcal{F}$ and there exists a natural transformation $\tau_{2}$ from $\mathcal{F}$ to $\mathcal{F}_{2}$ such that $f_{1}=\left\langle\left\langle\mathcal{F}, \mathcal{F}_{2}\right\rangle, \tau_{2}\right\rangle$ and $f_{2}=\left\langle\left\langle\mathcal{F}_{1}, \mathcal{F}\right\rangle, \tau_{1}\right\rangle$ and $f_{1} \circ f_{2}=\left\langle\left\langle\mathcal{F}_{1}\right.\right.$, $\left.\left.\mathcal{F}_{2}\right\rangle, \tau_{2}{ }^{\circ} \tau_{1}\right\rangle$ and for every morphisms $g_{1}, g_{2}$ of $\mathscr{C}_{1}$ such that $g_{2} \triangleright g_{1}$ holds $\tau_{2}\left(g_{2}\right) \triangleright \tau_{1}\left(g_{1}\right)$ and $\left(\tau_{2} \circ \tau_{1}\right)\left(g_{2} \circ g_{1}\right)=\tau_{2}\left(g_{2}\right) \circ \tau_{1}\left(g_{1}\right)$.

PRoOF: If $f_{1} \triangleright f_{2}$, then there exist covariant functors $\mathcal{F}, \mathcal{F}_{1}, \mathcal{F}_{2}$ from $\mathscr{C}_{1}$ to $\mathscr{C}_{2}$ and there exists a natural transformation $\tau_{1}$ from $\mathcal{F}_{1}$ to $\mathcal{F}$ and there exists a natural transformation $\tau_{2}$ from $\mathcal{F}$ to $\mathcal{F}_{2}$ such that $f_{1}=\langle\langle\mathcal{F}$, $\left.\left.\mathcal{F}_{2}\right\rangle, \tau_{2}\right\rangle$ and $f_{2}=\left\langle\left\langle\mathcal{F}_{1}, \mathcal{F}\right\rangle, \tau_{1}\right\rangle$ and $f_{1} \circ f_{2}=\left\langle\left\langle\mathcal{F}_{1}, \mathcal{F}_{2}\right\rangle, \tau_{2}{ }^{\circ} \tau_{1}\right\rangle$ and for every morphisms $g_{1}, g_{2}$ of $\mathscr{C}_{1}$ such that $g_{2} \triangleright g_{1}$ holds $\tau_{2}\left(g_{2}\right) \triangleright \tau_{1}\left(g_{1}\right)$ and $\left(\tau_{2}^{\circ} \tau_{1}\right)\left(g_{2} \circ g_{1}\right)=\tau_{2}\left(g_{2}\right) \circ \tau_{1}\left(g_{1}\right)$ by [6, (1)], (5), (58), [16, (5)].

Let us consider non empty categories $\mathscr{C}_{1}, \mathscr{C}_{2}$ and a morphism $f$ of Functors $\left(\mathscr{C}_{2}\right.$, $\left.\mathscr{C}_{1}\right)$. Now we state the propositions:

(64) $f$ is identity if and only if there exists a covariant functor $\mathcal{F}$ from $\mathscr{C}_{1}$ to $\mathscr{C}_{2}$ such that $f=\langle\langle\mathcal{F}, \mathcal{F}\rangle, \mathcal{F}\rangle$.

Proof: Set $\mathscr{C}=\operatorname{Functors}\left(\mathscr{C}_{2}, \mathscr{C}_{1}\right)$. If $f$ is identity, then there exists a covariant functor $\mathcal{F}$ from $\mathscr{C}_{1}$ to $\mathscr{C}_{2}$ such that $f=\langle\langle\mathcal{F}, \mathcal{F}\rangle, \mathcal{F}\rangle$ by [15, (24)], (63), (61), (5). Consider $\mathcal{F}$ being a covariant functor from $\mathscr{C}_{1}$ to $\mathscr{C}_{2}$ such that $f=\langle\langle\mathcal{F}, \mathcal{F}\rangle, \mathcal{F}\rangle$. For every morphism $f_{1}$ of $\mathscr{C}$ such that $f \triangleright f_{1}$ holds $f \circ f_{1}=f_{1}$ by (63), (5), (4), [7, (12)].

(65) There exist covariant functors $\mathcal{F}_{1}, \mathcal{F}_{2}$ from $\mathscr{C}_{1}$ to $\mathscr{C}_{2}$ and there exists a natural transformation $\tau$ from $\mathcal{F}_{1}$ to $\mathcal{F}_{2}$ such that $f=\left\langle\left\langle\mathcal{F}_{1}, \mathcal{F}_{2}\right\rangle, \tau\right\rangle$ and $\operatorname{dom} f=\left\langle\left\langle\mathcal{F}_{1}, \mathcal{F}_{1}\right\rangle, \mathcal{F}_{1}\right\rangle$ and $\operatorname{cod} f=\left\langle\left\langle\mathcal{F}_{2}, \mathcal{F}_{2}\right\rangle, \mathcal{F}_{2}\right\rangle$. The theorem is a consequence of (63) and (64). 


\section{Exponential ObJects}

Let $\mathscr{C}$ be a category with binary products, $a, b, c$ be objects of $\mathscr{C}$, and $e$ be a morphism from $c \times a$ to $b$. Assume $\operatorname{hom}(c \times a, b) \neq \emptyset$. We say that $\langle c, e\rangle$ is an exponent of $a$ and $b$ if and only if

(Def. 29) for every object $d$ of $\mathscr{C}$ and for every morphism $f$ from $d \times a$ to $b$ such that $\operatorname{hom}(d \times a, b) \neq \emptyset \operatorname{holds} \operatorname{hom}(d, c) \neq \emptyset$ and there exists a morphism $h$ from $d$ to $c$ such that $f=e \cdot(h \times \mathrm{id}-a)$ and for every morphism $h_{1}$ from $d$ to $c$ such that $f=e \cdot\left(h_{1} \times\right.$ id- $\left.a\right)$ holds $h=h_{1}$.

Now we state the propositions:

(66) Let us consider a category $\mathscr{C}$ with binary products, objects $a_{1}, a_{2}, b_{1}$, $b_{2}, c_{1}, c_{2}$ of $\mathscr{C}$, a morphism $f_{1}$ from $a_{1}$ to $b_{1}$, a morphism $f_{2}$ from $a_{2}$ to $b_{2}$, a morphism $g_{1}$ from $b_{1}$ to $c_{1}$, and a morphism $g_{2}$ from $b_{2}$ to $c_{2}$. Suppose $\operatorname{hom}\left(a_{1}, b_{1}\right) \neq \emptyset$ and $\operatorname{hom}\left(b_{1}, c_{1}\right) \neq \emptyset$ and $\operatorname{hom}\left(a_{2}, b_{2}\right) \neq \emptyset$ and $\operatorname{hom}\left(b_{2}, c_{2}\right) \neq \emptyset$. Then $\left(g_{1} \times g_{2}\right) \cdot\left(f_{1} \times f_{2}\right)=g_{1} \cdot f_{1} \times\left(g_{2} \cdot f_{2}\right)$. The theorem is a consequence of (42) and (44).

(67) Let us consider a category $\mathscr{C}$ with binary products, and objects $a, b$ of $\mathscr{C}$. Then id- $a \times \operatorname{id}-b=\operatorname{id}-(a \times b)$. The theorem is a consequence of (42).

(68) Let us consider a category $\mathscr{C}$ with binary products, objects $a, b, c_{1}, c_{2}$ of $\mathscr{C}$, a morphism $e_{1}$ from $c_{1} \times a$ to $b$, and a morphism $e_{2}$ from $c_{2} \times a$ to b. Suppose $\operatorname{hom}\left(c_{1} \times a, b\right) \neq \emptyset$ and $\operatorname{hom}\left(c_{2} \times a, b\right) \neq \emptyset$ and $\left\langle c_{1}, e_{1}\right\rangle$ is an exponent of $a$ and $b$ and $\left\langle c_{2}, e_{2}\right\rangle$ is an exponent of $a$ and $b$. Then $c_{1}$ and $c_{2}$ are isomorphic.

Proof: There exists a morphism $f$ from $c_{1}$ to $c_{2}$ such that $f$ is isomorphism by (44), [16, (23)], (66), [16, (18)].

Let $\mathscr{C}$ be a category with binary products. We say that $\mathscr{C}$ has exponential objects if and only if

(Def. 30) for every objects $a, b$ of $\mathscr{C}$, there exists an object $c$ of $\mathscr{C}$ and there exists a morphism $e$ from $c \times a$ to $b$ such that $\operatorname{hom}(c \times a, b) \neq \emptyset$ and $\langle c, e\rangle$ is an exponent of $a$ and $b$.

One can check that $\mathbf{1}$ has binary products.

Now we state the proposition:

(69) 1 has exponential objects.

Proof: Set $\mathscr{C}=\mathbf{1}$. Consider $f$ being a morphism of $\mathbf{1}$ such that $f$ is identity and $\mathrm{Ob} \mathbf{1}=\{f\}$ and Mor $\mathbf{1}=\{f\}$. For every objects $o_{1}, o_{2}$ of $\mathscr{C}$, every morphism of $\mathscr{C}$ is a morphism from $o_{1}$ to $o_{2}$ by [16, (20)]. For every objects $a, b$ of $\mathscr{C}$, there exists an object $c$ of $\mathscr{C}$ and there exists a morphism $e$ from $c \times a$ to $b$ such that $\operatorname{hom}(c \times a, b) \neq \emptyset$ and $\langle c, e\rangle$ is an exponent of $a$ and $b$. 
Let us observe that there exists a category with binary products which has exponential objects.

Let $\mathscr{C}$ be a category with exponential objects binary products and $a, b$ be objects of $\mathscr{C}$.

A categorical exponent of $a$ and $b$ is a pair object and is defined by

(Def. 31) there exists an object $c$ of $\mathscr{C}$ and there exists a morphism $e$ from $c \times a$ to $b$ such that $i t=\langle c, e\rangle$ and $\operatorname{hom}(c \times a, b) \neq \emptyset$ and $\langle c, e\rangle$ is an exponent of $a$ and $b$.

The functor $b^{a}$ yielding an object of $\mathscr{C}$ is defined by the term

(Def. 32) (the categorical exponent of $a$ and $b)_{\mathbf{1}}$.

The functor eval $(a, b)$ yielding a morphism from $b^{a} \times a$ to $b$ is defined by the term

(Def. 33) (the categorical exponent of $a$ and $b)_{\mathbf{2}}$.

Now we state the propositions:

(70) Let us consider a category $\mathscr{C}$ with exponential objects binary products, and objects $a, b$ of $\mathscr{C}$. Then

(i) $\operatorname{hom}\left(b^{a} \times a, b\right) \neq \emptyset$, and

(ii) $\left\langle b^{a}, \operatorname{eval}(a, b)\right\rangle$ is an exponent of $a$ and $b$.

(71) Let us consider a category $\mathscr{C}$ with exponential objects binary products, and objects $a, b, c$ of $\mathscr{C}$. Suppose $\operatorname{hom}(c \times a, b) \neq \emptyset$. Then there exists a function $L$ from $\operatorname{hom}(c \times a, b)$ into $\operatorname{hom}\left(c, b^{a}\right)$ such that

(i) for every morphism $f$ from $c \times a$ to $b$ and for every morphism $h$ from $c$ to $b^{a}$ such that $h=L(f) \operatorname{holds} \operatorname{eval}(a, b) \cdot(h \times \mathrm{id}-a)=f$, and

(ii) $L$ is bijective.

Proof: $\operatorname{hom}\left(b^{a} \times a, b\right) \neq \emptyset$ and $\left\langle b^{a}, \operatorname{eval}(a, b)\right\rangle$ is an exponent of $a$ and $b$. Define $\mathcal{P}$ [object, object] $\equiv$ for every morphism $f$ from $c \times a$ to $b$ such that $f=\$_{1}$ there exists a morphism $h$ from $c$ to $b^{a}$ such that $h=\$_{2}$ and $f=\operatorname{eval}(a, b) \cdot(h \times \mathrm{id}-a)$ and for every morphism $h_{1}$ from $c$ to $b^{a}$ such that $f=\operatorname{eval}(a, b) \cdot\left(h_{1} \times\right.$ id- $\left.a\right)$ holds $h=h_{1}$. For every object $x$ such that $x \in \operatorname{hom}(c \times a, b)$ there exists an object $y$ such that $y \in \operatorname{hom}\left(c, b^{a}\right)$ and $\mathcal{P}[x, y]$. Consider $L$ being a function from $\operatorname{hom}(c \times a, b)$ into $\operatorname{hom}\left(c, b^{a}\right)$ such that for every object $x$ such that $x \in \operatorname{hom}(c \times a, b)$ holds $\mathcal{P}[x, L(x)]$ from [7, Sch. 1]. There exists an object $y$ such that $y \in \operatorname{hom}\left(c, b^{a}\right)$. For every morphism $f$ from $c \times a$ to $b$ and for every morphism $h$ from $c$ to $b^{a}$ such that $h=L(f)$ holds $\operatorname{eval}(a, b) \cdot(h \times$ id- $a)=f$. For every objects $x_{1}$, $x_{2}$ such that $x_{1}, x_{2} \in \operatorname{hom}(c \times a, b)$ and $L\left(x_{1}\right)=L\left(x_{2}\right)$ holds $x_{1}=x_{2}$. For every object $y$ such that $y \in \operatorname{hom}\left(c, b^{a}\right)$ holds $y \in \operatorname{rng} L$ by [6. (3)]. 
Let $\mathscr{A}, \mathscr{B}, \mathscr{C}$ be categories and $\mathcal{E}$ be a functor from $\mathscr{C} \times \mathscr{A}$ to $\mathscr{B}$. Assume $\mathcal{E}$ is covariant. We say that $\langle\mathscr{C}, \mathcal{E}\rangle$ is an exponent of $\mathscr{A}$ and $\mathscr{B}$ if and only if

(Def. 34) for every category $\mathscr{D}$ and for every functor $\mathcal{F}$ from $\mathscr{D} \times \mathscr{A}$ to $\mathscr{B}$ such that $\mathcal{F}$ is covariant there exists a functor $\mathcal{H}$ from $\mathscr{D}$ to $\mathscr{C}$ such that $\mathcal{H}$ is covariant and $\mathcal{F}=\mathcal{E} \circ\left(\mathcal{H} \times \operatorname{id}_{\mathscr{A}}\right)$ and for every functor $\mathcal{H}_{1}$ from $\mathscr{D}$ to $\mathscr{C}$ such that $\mathcal{H}_{1}$ is covariant and $\mathcal{F}=\mathcal{E} \circ\left(\mathcal{H}_{1} \times \operatorname{id}_{\mathscr{A}}\right)$ holds $\mathcal{H}=\mathcal{H}_{1}$.

Let $\mathscr{C}_{1}, \mathscr{C}_{2}$ be categories.

A categorical exponent of $\mathscr{C}_{1}$ and $\mathscr{C}_{2}$ is a pair object and is defined by

(Def. 35) there exists a category $\mathscr{C}$ and there exists a functor $\mathcal{E}$ from $\mathscr{C} \times \mathscr{C}_{1}$ to $\mathscr{C}_{2}$ such that $i t=\langle\mathscr{C}, \mathcal{E}\rangle$ and $\mathcal{E}$ is covariant and $\langle\mathscr{C}, \mathcal{E}\rangle$ is an exponent of $\mathscr{C}_{1}$ and $\mathscr{C}_{2}$.

The functor $\mathscr{C}_{2}^{\mathscr{C}_{1}}$ yielding a category is defined by the term

(Def. 36) (the categorical exponent of $\mathscr{C}_{1}$ and $\left.\mathscr{C}_{2}\right)_{\mathbf{1}}$.

The functor eval $\left(\mathscr{C}_{1}, \mathscr{C}_{2}\right)$ yielding a functor from $\mathscr{C}_{2} \mathscr{C}_{1} \times \mathscr{C}_{1}$ to $\mathscr{C}_{2}$ is defined by the term

(Def. 37) (the categorical exponent of $\mathscr{C}_{1}$ and $\left.\mathscr{C}_{2}\right)_{\mathbf{2}}$.

Now we state the propositions:

(72) Let us consider categories $\mathscr{C}_{1}, \mathscr{C}_{2}$. Then $\left\langle\mathscr{C}_{2} \mathscr{C}_{1}\right.$, eval $\left.\left(\mathscr{C}_{1}, \mathscr{C}_{2}\right)\right\rangle$ is an exponent of $\mathscr{C}_{1}$ and $\mathscr{C}_{2}$.

(73) Let us consider categories $\mathscr{A}, \mathscr{B}, \mathscr{C}_{1}, \mathscr{C}_{2}$, a functor $\mathcal{E}_{1}$ from $\mathscr{C}_{1} \times \mathscr{A}$ to $\mathscr{B}$, and a functor $\mathcal{E}_{2}$ from $\mathscr{C}_{2} \times \mathscr{A}$ to $\mathscr{B}$. Suppose $\mathcal{E}_{1}$ is covariant and $\mathcal{E}_{2}$ is covariant and $\left\langle\mathscr{C}_{1}, \mathcal{E}_{1}\right\rangle$ is an exponent of $\mathscr{A}$ and $\mathscr{B}$ and $\left\langle\mathscr{C}_{2}, \mathcal{E}_{2}\right\rangle$ is an exponent of $\mathscr{A}$ and $\mathscr{B}$. Then $\mathscr{C}_{1} \cong \mathscr{C}_{2}$.

Proof: There exists a functor $\mathcal{F}$ from $\mathscr{C}_{1}$ to $\mathscr{C}_{2}$ and there exists a functor $\mathcal{G}$ from $\mathscr{C}_{2}$ to $\mathscr{C}_{1}$ such that $\mathcal{F}$ is covariant and $\mathcal{G}$ is covariant and $\mathcal{G} \circ \mathcal{F}=\operatorname{id}_{\mathscr{C}_{1}}$ and $\mathcal{F} \circ \mathcal{G}=\operatorname{id}_{\mathscr{C}_{2}}$ by [16, (10)], (50), [16, (11)], [15, (35)].

Let $\mathscr{C}_{1}, \mathscr{C}_{2}$ be categories. Observe that eval $\left(\mathscr{C}_{1}, \mathscr{C}_{2}\right)$ is covariant.

Let $\mathscr{C}_{1}$ be a non empty category and $\mathscr{C}_{2}$ be an empty category. Let us note that $\mathscr{C}_{2} \mathscr{C}_{1}$ is empty.

Let $\mathscr{C}_{1}$ be an empty category and $\mathscr{C}_{2}$ be a category. Let us observe that $\mathscr{C}_{2} \mathscr{C}_{1}$ is non empty and trivial.

Let $\mathscr{C}_{1}$ be a non empty category and $\mathscr{C}_{2}$ be a non empty category. One can verify that $\mathscr{C}_{2} \mathscr{C}_{1}$ is non empty.

Now we state the proposition:

(74) Let us consider categories $\mathscr{C}_{1}, \mathscr{C}_{2}$. Then Functors $\left(\mathscr{C}_{2}, \mathscr{C}_{1}\right) \cong \mathscr{C}_{2} \mathscr{C}_{1}$. The theorem is a consequence of $(28),(72)$, and (73). 


\section{REFERENCES}

[1] Jiri Adamek, Horst Herrlich, and George E. Strecker. Abstract and Concrete Categories: The Joy of Cats. Dover Publication, New York, 2009.

[2] Grzegorz Bancerek. Cardinal numbers Formalized Mathematics, 1(2):377-382, 1990.

[3] Grzegorz Bancerek. The ordinal numbers Formalized Mathematics, 1(1):91-96, 1990.

[4] Francis Borceaux. Handbook of Categorical Algebra I. Basic Category Theory, volume 50 of Encyclopedia of Mathematics and its Applications. Cambridge University Press, Cambridge, 1994.

[5] Czesław Byliński. Introduction to categories and functors Formalized Mathematics, 1 (2):409-420, 1990.

[6] Czesław Byliński. Functions and their basic properties Formalized Mathematics, 1(1): $55-65,1990$.

[7] Czesław Byliński. Functions from a set to a set Formalized Mathematics, 1(1):153-164, 1990.

[8] Czesław Byliński. Partial functions. Formalized Mathematics, 1(2):357-367, 1990.

[9] Czesław Byliński. Some basic properties of sets Formalized Mathematics, 1(1):47-53, 1990.

[10] Agata Darmochwał. Finite sets Formalized Mathematics, 1(1):165-167, 1990.

[11] Krzysztof Hryniewiecki. Graphs. Formalized Mathematics, 2(3):365-370, 1991.

[12] F. William Lawvere. Functorial semantics of algebraic theories and some algebraic problems in the context of functorial semantics of algebraic theories. Reprints in Theory and Applications of Categories, 5:1-121, 2004.

[13] Saunders Mac Lane. Categories for the Working Mathematician, volume 5 of Graduate Texts in Mathematics. Springer Verlag, New York, Heidelberg, Berlin, 1971.

[14] Beata Padlewska. Families of sets Formalized Mathematics, 1(1):147-152, 1990.

[15] Marco Riccardi. Obiect-free definition of categories. Formalized Mathematics, 21(3): 193-205, 2013. doi $10.2478 /$ forma-2013-0021.

[16] Marco Riccardi. Categorical pullbacks. Formalized Mathematics, 23(1):1-14, 2015. doi:10.2478/forma-2015-0001

[17] Andrzej Trybulec. Enumerated sets. Formalized Mathematics, 1(1):25-34, 1990.

[18] Andrzej Trybulec. Isomorphisms of categories Formalized Mathematics, 2(5):629-634, 1991.

[19] Andrzej Trybulec. Natural transformations. Discrete categories. Formalized Mathematics, 2(4):467-474, 1991.

[20] Zinaida Trybulec. Properties of subsets Formalized Mathematics, 1(1):67-71, 1990.

[21] Edmund Woronowicz. Relations and their basic properties. Formalized Mathematics, 1 (1):73-83, 1990.

[22] Edmund Woronowicz. Relations defined on sets. Formalized Mathematics, 1(1):181-186, 1990. 